\title{
Les chemins de fer secondaires d'Alsace, hier et demain
}

Joël Forthoffer

\section{OpenEdition}

\section{Journals}

Édition électronique

URL : https://journals.openedition.org/rhcf/2047

DOI : 10.4000/rhcf.2047

\section{Éditeur}

Rails \& histoire

\section{Édition imprimée}

Date de publication : 2 mai 2002

Pagination : 190-210

ISBN : 00996-9403

ISSN : 0996-9403

\section{Référence électronique}

Joël Forthoffer, «Les chemins de fer secondaires d'Alsace, hier et demain », Revue d'histoire des chemins de fer [En ligne], 24-25 | 2002, mis en ligne le 14 avril 2015, consulté le 22 avril 2022. URL http://journals.openedition.org/rhcf/2047 ; DOI : https://doi.org/10.4000/rhcf.2047 


\section{Joël FORTHOFFER}

\section{Les chemins de fer secondaires d'Alsace, hier et demain}

Cette approche n'a pour prétention que d'apporter un éclairage sur l'évolution du réseau de chemins de fer en Alsace. Une étude purement descriptive des chemins de fer n'aurait guère de signification, ni d'utilité. Elle ne prend de sens et de valeur que replacée dans son contexte géographique, historique, économique et humain. Sans la connaissance du contexte dans lequel ce mode de transport inscrit son activité, elle peut s'apprendre, mais non se comprendre.

Bien que tout découpage soit arbitraire, nous distinguerons, des origines à nos jours ${ }^{1}$, quatre périodes :

- une phase de construction et d'expansion, des origines à 1870, au cours de laquelle le chemin de fer jouit d'un monopole de fait ;

- une phase de développement pendant la période allemande de 1870 jusqu'à la fin de la Première Guerre mondiale, au cours de laquelle le chemin de fer est adapté aux nouvelles conditions économiques du Reich allemand ;

- pendant l'entre-deux-guerres, de 1918 à la fin de la Seconde Guerre mondiale, l'apparition de la concurrence routière aggrave la gestion déjà difficile des réseaux secondaires et provoque la disparition de beaucoup d'entre eux ;

- avec l'après-guerre vient la reconstruction, puis le renouveau du transport ferroviaire avec l'apparition de la régionalisation.

Les cartes suivantes (fig. 1) illustrent les principales évolutions du réseau ferré sur la région Est. L'ensemble des voies ferrées construites en Alsace est représenté par la carte en annexe où figure également la chronologie de l'ouverture des lignes.

Naissance et développement du réseau ferré, des origines à 1870

Jusqu'en 1842, les lignes construites sont essentiellement des lignes d'intérêt local, au sens propre du terme. C'est l'époque des tronçons et des initiatives locales comme les lignes Thann-Mulhouse et StrasbourgBâle. Cependant, Legrand et les saint-simoniens conçoivent déjà l'armature du réseau national. Les projets défendus devant le Parlement 


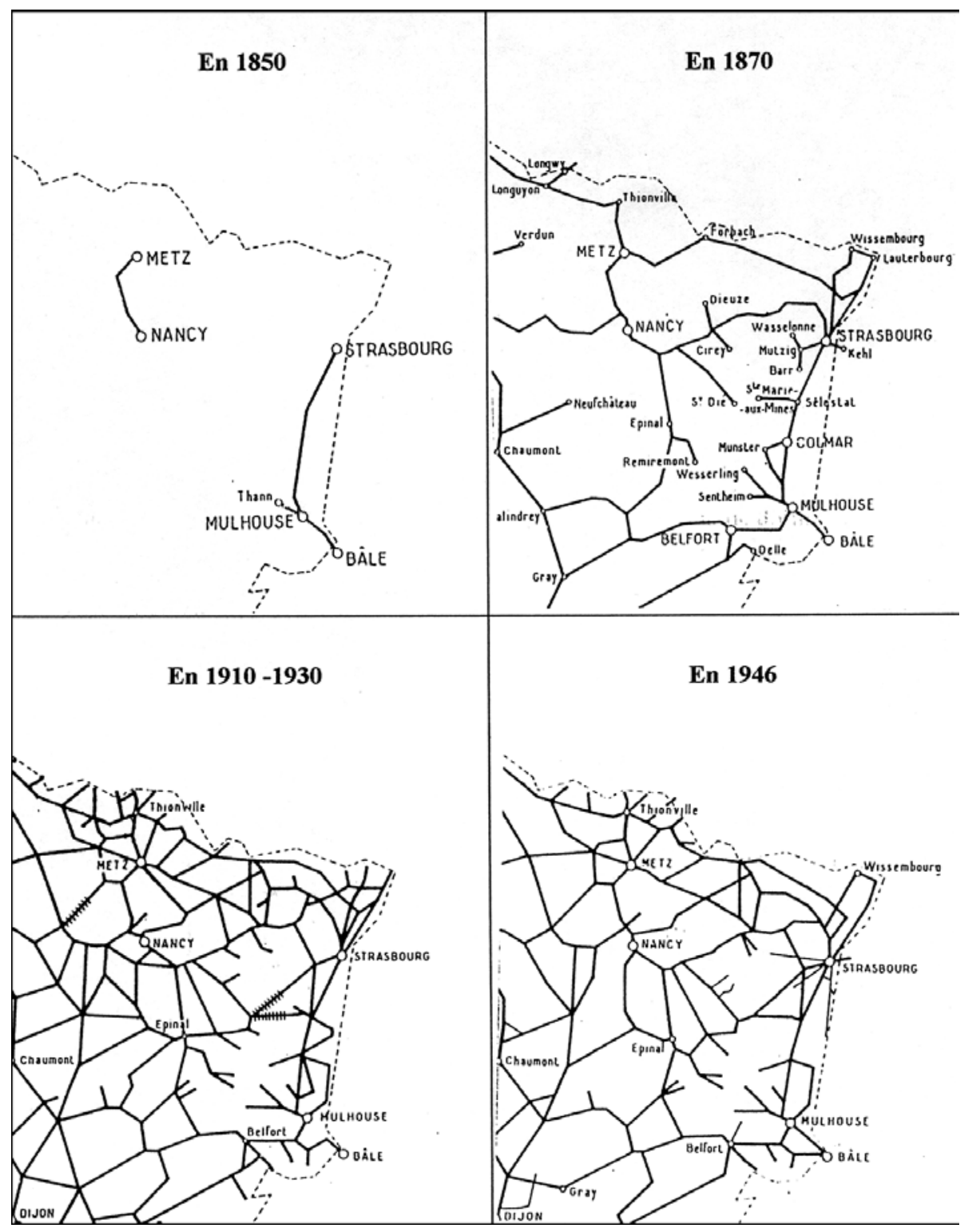

Figure 1 : Évolution du réseau ferré alsacien.

$\odot_{\text {J. Forthoffer }}$ 
aboutissent à la loi du 11 juillet 1842 qui constitue en quelque sorte, on l'a dit, la Charte du chemin de fer. Les liaisons Paris-Bâle et ParisStrasbourg font partie du programme de construction. Mais l'essor du chemin de fer est stoppé de 1846 à 1850 par une crise économique et par la Révolution de 1848.

Les chemins de fer secondaires sont souvent exploités par une société anonyme à capitaux privés pour le compte d'une collectivité (ville, département...), comme c'est le cas pour la ligne Mulhouse-Thann. Conçue dans un but industriel pour diminuer le coût du transport des matières premières, elle connait d'abord un grand succès avec le transport de voyageurs : 10400 personnes sont transportées en janvier 1840 et, pour les six premiers mois de la même année, la moyenne journalière s'élève à 680 voyageurs. Quant au trafic marchandises, il ne démarre qu'en juillet 1840, et ne dépassera guère le stade régional, avec des recettes qui ne représentent que près de $12 \%$ de celles du trafic voyageurs. Cette première ligne de chemin de fer se révélait parfaitement fiable et rentable. De plus, elle prouvait que la traction par locomotive à vapeur était possible.

Une période de prospérité économique croissante accompagne l'Empire jusqu'en 1870. Elle est marquée par un développement considérable du réseau d'intérêt général et par la naissance du réseau d'intérêt local. De nombreuses industries : forges (De Dietrich), filatures (Hartmann), textiles, ainsi que la population ouvrière souhaitent la création de nouvelles lignes ferroviaires. Cette double évolution répond aux conceptions politiques comme économiques de Napoléon III, partisan d'une centralisation administrative mais favorable au libéralisme économique.

L'évolution de la technique ferroviaire a permis le développement des transports urbains et campagnards dotés d'un matériel léger. Les chemins de fer secondaires ont grandement contribué au désenclavement du milieu rural. Ils emmenaient les voyageurs vers le chef-lieu de canton ou la ville préfecture pour leurs nécessités ou leurs plaisirs. De temps à autre, un voyage plus lointain, vers une capitale, était envisagé.

L'essor des chemins de fer porte un sérieux préjudice à la navigation fluviale dont le trafic fléchit de près du quart de 1847 à 1870.

En 1855, le préfet du Bas-Rhin, Jean-Baptiste Migneret, fut le premier en France à donner à son département un réseau ferré d'intérêt local particulier.

Son projet repose sur l'idée d'utiliser les chemins vicinaux et routes départementales pour y construire des lignes de chemins de fer. C'est l'ingénieur Coumes qui réunit tous les éléments pour atteindre le but 
assigné et rendre la réalisation démonstrative. Il applique à l'exécution de la plate-forme d'un réseau départemental les ressources de la vicinalité et les dispositions de la loi du 21 mai 1836. Les travaux ont été réalisés aussi"économiquement que possible une fois les terrains acquis. Ainsi, un an avant la loi du 12 juillet 1865 relative aux chemins de fer d'intérêt local, le département du Bas-Rhin inaugure le 29 septembre 1864 la ligne Strasbourg-Barr, le 15 décembre celle de Molsheim à Mutzig et à Wasselonne et, le 18 décembre, celle de Niederbronn à Haguenau.

Les trois lignes de Strasbourg à Barr, Mutzig et Wasselonne, de Niederbronn à Haguenau et de Séléstat à Sainte-Marie-aux-Mines, totalisant une longueur de $90 \mathrm{~km}$, ont été ouvertes sans que la dépense dépasse $117300 \mathrm{~F}$ par kilomètre, matériel roulant compris.

L'initiative de grands industriels et du préfet transforme les chemins vicinaux qui relient de nombreuses usines en chemins vicinaux ferrés et les fait aboutir au chef-lieu ou aux gares de grandes lignes. Un réseau naît: «En partant de ce point [...] et en jetant les yeux sur le département du Bas-Rhin, on remarque que, fermé à l'Ouest par la chaîne des Vosges et à l'Est par le Rhin, il est parcouru du Nord au Sud, dans toute sa longueur, par une voie de fer communiquant avec l'Allemagne et tout le midi de la France, et, de l'Est à l'Ouest, par une voie croisant la première et communiquant avec la France septentrionnale d'un côté et l'Allemagne orientale de l'autre. On remarque enfin que, sur 33 cantons qui le composent, 16 sont traversés par ces chemins ${ }^{2}$.»

Le but est de multiplier les dessertes ferroviaires sans être obligé de faire face au prix élevé d'exploitation du kilomètre de grande ligne.

Le rapport de 1858 du préfet Migneret précise :

« la Compagnie des Chemins de Fer de l'Est est l'unique concessionnaire des voies régionales et en assure l'exploitation. [...]

De 1836 à aujourd'hui, le progrès a consisté à élever la vicinalité à la hauteur des routes nationales; de 1864 à une date quelconque de l'avenir, mais qui arrivera certainement, il consistera à élever la vicinalité à la hauteur des voies ferrées ${ }^{3}$.»

Le gouvernement français comprit rapidement qu'il y avait là une idée juste et pratique qui devait se généraliser. Il s'occupe de donner à son exécution une base légale avec la loi du 12 juillet 1865. Le département du Haut-Rhin et la Sarthe suivent alors l'exemple du Bas-Rhin.

L'action du préfet n'a pas permis de créer un chemin de fer départemental au même titre qu'un réseau routier départemental, mais elle a quand même donné l'impulsion qui entraîna la mise en service des lignes vicinales précédemment citées ainsi que celles de : Thann à Wesserling le 25 novembre 1863, prolongement de la ligne de Niederbronn jusqu'à 
Sarreguemines le 8 décembre 1869 et, enfin, celle de Bollwiller à Guebwiller le 5 février 1870. Aussi, à la veille de la guerre, l'implantation du chemin de fer apparaissait comme une véritable réussite. Le tissu économique en Alsace s'est trouvé transformé avec l'essor des industries mécaniques, notamment dans le domaine de la construction de locomotives.

\section{Développement du réseau pendant la période allemande (1870-1918)}

Les événements de 1870-1871 obligent au remaniement du réseau français par suite de la cession de l'Alsace et d'une partie de la Lorraine à l'Allemagne. Dès l'occupation des territoires par les troupes allemandes, la reprise du trafic sur les lignes est confiée à des commissions militaires d'exploitation. Pour l'Alsace et la Lorraine des commissions sont en place à Strasbourg et à Sarrebruck avec la mission impérative de rétablir les interceptions des voies puis de gérer les lignes conquises afin de satisfaire en priorité les besoins militaires. Ainsi, trois jours après la bataille de Wörth ( 9 août 1870), des trains circulent à nouveau de la frontière du Palatinat jusqu'à Haguenau, puis le 14 août jusqu'à Vendenheim. Le service est rétabli dans la plaine d'Alsace au gré des combats et définitivement entre Strasbourg et Mulhouse le 14 novembre 1870. Sur le pont de Kehl, dont les travées tournantes ont été détruites, la marche des trains reprend le 15 novembre 1870.

Pendant les hostilités, la commission de Strasbourg fait même poser, pour des raisons stratégiques, la seconde voie de la section Wissembourg-Vendenheim.

Dès avant le traité de Francfort, des conventions particulières sont passées entre l'administration allemande et la Compagnie de l'Est, et le service des trains reprend le 8 avril 1871 au-delà de Mulhouse vers Belfort. Le 10 mai 1871, en vertu du traité de Francfort, les chemins de fer établis en Alsace et en Lorraine sont cédés à l'empire allemand, moyennant une indemnité globale et forfaitaire de rachat équivalant à $325000000 \mathrm{~F}$, charge restant au gouvernement français de désintéresser la Compagnie de l'Est. La Kaiserliche Generaldirektion der Eisenbahn in Elsaß-Lothringen (E.L.) est créée sept mois plus tard.

Après la reconstruction de l'infrastructure, la direction de Strasbourg se préoccupe d'assurer l'extension des lignes intérieures et aussi l'amélioration des liaisons directes des villes importantes d'Alsace et de Lorraine avec l'Allemagne. Le premier objectif consistait à garantir l'approvisionnement en charbon depuis la Sarre pour encourager le développement industriel de la rive droite du Rhin, d'où la création d'une section de ligne transversale : Bouxwiller-Haguenau et HaguenauRoppenheim (1895), ainsi que des passages sur le Rhin à Roppenheim (1895), 
Neuf Brisach (1878) et Neuenbourg (1878). Les routes de campagne de l'époque étaient étroites et de faible débit, leur revêtement de cailloux ne résistait pas à une circulation intense. Les convois hippomobiles ne pouvaient transporter que peu de matériel à la fois, un poids limité, avec une faible vitesse. Aussi, le réseau des chemins de fer vicinaux est-il développé par les Allemands qui l'ont englobé dans leur réseau stratégique en doublant la plupart des voies comme entre Strasbourg et Mutzig.

Les chemins de fer à voie étroite auraient certainement pris un développement considérable en Alsace, si le réseau à voie normale y avait été moins dense. Cependant, il a été créé plus de $150 \mathrm{~km}$ de ces lignes. A partir de 1870, les Allemands complètent le réseau par un réseau à voie étroite centré autour de Colmar :

- Colmar-Kaysersberg-Lapoutroie-Le Bonhomme,

- Colmar-Wintzenheim,

- Colmar-Ensisheim relié au tramway de Mulhouse,

- Colmar-Markolsheim-Sundhoffen,

- Colmar-Schnierlach (Lapoutroie)-Rhinau-Strasbourg.

Il faut y ajouter la ligne touristique Munster-La Schlucht où elle rencontre la ligne Remiremont-Gérardmer-Le Hohneck ce qui permet de franchir les Vosges. Le site de Trois-Épis est desservi par un chemin de fer électrique depuis Turckheim avec un parcours de $7,8 \mathrm{~km}$ en montagne (dénivelé $400 \mathrm{~m}$ ). Inaugurée en 1894, la ligne a été construite pour le transport de pèlerins.

De ces chemins de fer à voie étroite, les uns appartiennent à l'État, les autres à des compagnies privées.

Les chemins de fer dépendant de l'État sont:

- la ligne de Lutzelbourg à Phalsbourg, créée en 1883, longue de $6 \mathrm{~km}$, et se reliant à Lutzelbourg avec la ligne Strasbourg-Sarrebourg.

- La ligne de Colmar à Markolsheim, comprenant les sections Colmar-Horbourg, ouverte en 1885, et Horbourg-Markolsheim, en 1890 ; longue de $22 \mathrm{~km}$, elle est reliée, à Colmar, aux lignes StrasbourgBâle et Colmar-Brisach, et se raccorde, à Marckolsheim, avec la ligne des tramways strasbourgeois, à Colmar, avec les lignes ColmarLapoutroie et Colmar-Wintzenheim.

Les lignes de chemins de fer appartenant à des compagnies privées sont :

- la ligne de Rosheim à Ottrot : longue de 8 kilomètres, elle se détache de la ligne Molsheim-Barr à la gare de Rosheim pour aboutir à Ottrot via Boersch.

- Les tramways strasbourgeois, complétés par de longues antennes rurales comme la section Strasbourg-Marckolsheim de 54,5 km, qui circulent d'abord à vapeur, puis sont électrifiés. 

$(7,5 \mathrm{~km})$.

- Les tramways de Mulhouse à Ensisheim (16,5 km) et Wittenheim

- Les lignes de la vallée de Kaisersberg avec la section de Colmar à Wintzenheim (5 km) et Colmar-Lapoutroie $(25 \mathrm{~km})$, par la vallée de la Weiss.

La voie étroite permet des courbes de faible rayon sur lesquelles circule un matériel court et léger entraînant ainsi l'utilisation d'un rail moins pesant sur une infrastructure moins profonde. La voie métrique est toute désignée pour assurer le trafic faible et peu lourd d'une ligne secondaire. Elle réduit alors de moitié la plupart des postes de dépenses par rapport à la voie normale, sans compter l'économie des travaux d'infrastructure et ouvrages d'art.

Les tramways sont des chemins de fer à rails généralement plats, établis dans les rues des villes ou sur les accotements des routes et plus spécialement destinés au transport de voyageurs. Cependant, le cadre juridique des tramways est identique à celui des chemins de fer secondaires. Tels s'étaient construits les premiers tramways. Mais rapidement se construisent, sous le nom de tramway, de véritables chemins de fer d'intérêt local qui, comme ces derniers, relient les localités parfois très éloignées et transportent mêmes des marchandises. Ils ne se distinguent des chemins de fer que du point de vue de leur construction, en ne quittant pas les routes. Ainsi le réseau suburbain des tramways strasbourgeois relie Truchtersheim, Breuchswickersheim, Oberhausbergen, Westhoffen. La liaison Kochersberg-Strasbourg-Erstein permet l'acheminement des betteraves vers la sucrerie d'Erstein.

Au total, le réseau à voie étroite compte $180 \mathrm{~km}$ de voies métriques à la veille de la Première Guerre mondiale construites suivant des normes moins exigeantes que pour le réseau principal. A la veille du premier conflit mondial ce sont sept lignes qui relient le réseau à l'Allemagne et deux seulement à la France. Pendant la guerre de 1914-1918, les Allemands ont construit un chemin de fer militaire pour acheminer le matériel et les hommes sur la ligne tout près des tranchées. La « Lordonbahn » reliait ainsi Villé, par le col d’Urbais, à la vallée de Fave dans les Vosges où passait son front. La ligne construite avec un écartement de voie de $60 \mathrm{~cm}$ comptait quelques $42 \mathrm{~km}$ avec plus de 200 mètres de dénivelé (fig. 2).

Le nouveau tracé des frontières a donc modifié les limites de l'espace. Les activités économiques ont évolué et leur distribution géographique s'est transformée. Le réseau de transport ferroviaire s'est adapté à ce nouveau territoire et à ses besoins. On retiendra de cette période le désenclavement des campagnes avec le développement sans précédent du réseau comme en témoigne la chronologie des lignes de chemin de fer d'Alsace (en annexe). 


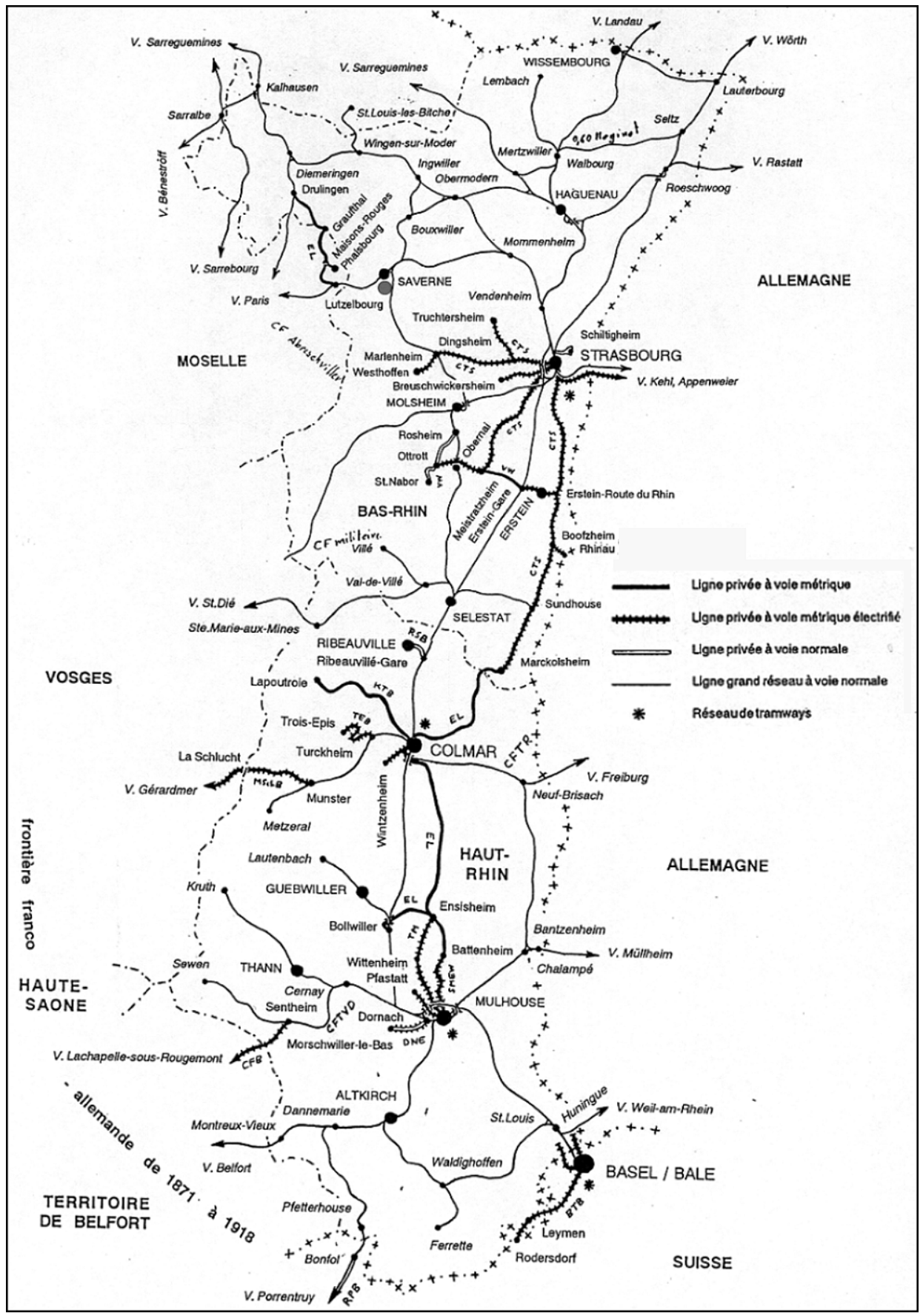

Source : A. Gibert, J. Cassando, Trains oubliés, vol. 1, L'Alsace-Lorraine - l'Est, Menton, Editions du Cabri, 1981.

Figure 2 : Récapitulation des lignes ferroviaires en Alsace des origines (1839) à 1950. 


\section{Stabilisation et adaptation du réseau (1918-1945)}

Les conséquences de la guerre et la mise en place du réseau d'Alsace et de Lorraine (A.L.) caractérisent la période d'avant-guerre jusqu'en 1937. Si les réseaux ont contribué de façon remarquable à la victoire de 1918, ils se sont trouvés, à la fin de la guerre, dans une situation extrêmement difficile : installations détruites, matériel en mauvais état, hausse des prix et des salaires...

Les chemins de fer A.L. sont constitués par un arrêté en date du 19 juin 1919 et un décret du 30 novembre 1920 place la nouvelle administration sous la tutelle directe du ministère des Travaux publics. La cession définitive est consacrée par l'article 67 du traité de Versailles signé le 28 juin 1919 : « Le gouvernement français est subrogé dans les droits de l'empire allemand sur toutes les lignes de chemin de fer gérées par l'administration des chemins de fer de l'empire et actuellement en exploitation ou en construction [...] Cette subrogation ne donnera lieu à la charge de l'État français à aucun paiement. » Dès la reprise du service sous l'autorité française de nombreuses questions se posent, dont les principales sont l'élimination progressive des agents de nationalité allemande par le recrutement et la formation de personnel et la remise en état des installations fixes endommagées par faits de guerre.

Les grandes compagnies signent avec l'État la convention du 28 juin 1921 qui marque un nouveau pas vers la concentration et la rationalisation ; cette convention ne comporte pas de concession de lignes nouvelles.

Suit une période de prospérité économique d'après-guerre qui se caractérise par une rénovation et un redressement des réseaux : plan d'électrification de 1920, modernisation des installations de sécurité et la construction en Alsace de six sections de lignes. La première ligne mise en service après la Première Guerre mondiale a été celle de Waldighofen à Saint-Louis, le $1^{\text {er }}$ mars 1920. Puis ont suivi : la section de ligne Schalbach-Drulingen (juillet 1922), la ligne NeufBrisach-Bantzenheim (avril 1923), la première percée des Vosges avec la ligne Saint-Dié à Saales (octobre 1928), la section MontagneVerte-Koenigshoffen et la deuxième percée des Vosges, Saint-Dié à Sainte-Marie-aux-Mines (août 1937). Le réseau A.L. est incorporé le $1^{\text {er }}$ janvier 1938 à la Région de l’Est de la SNCF.

Pendant ce temps nous assistons, en France, à la naissance et l'essor de la concurrence routière avec 80000 véhicules industriels en 1920 et 450000 en 1932. Quant à l'apparition de l'automobile, elle date de 1891, Panhard Levasseur met en vente la première voiture française et le nombre des véhicules passe rapidement de 91000 en 1913 à 860000 en 1925. Les caractéristiques de la circulation s'en trouvent profondément modifiées et un concurrent au chemin de fer nait. 


\section{Les lignes militaires : la voie de 60 de la Ligne Maginot}

Dès la fin de la Première Guerre mondiale, le gouvernement français veut à tout prix éviter la possibilité d'une nouvelle invasion semblable. En 1929, une conception d'ensemble de l'organisation défensive est arrêtée. Les travaux importants de la Ligne Maginot commencent en 1930. Les ouvrages les plus importants (fig. 3) sont raccordés à la voie de 60 et se situent en Alsace du Nord : Hochwald, Schoenenbourg et le Four-à-chaux (Lembach). A titre de comparaison, le rendement de la voie de 60 est estimé à 100 à 125 tonnes par heure alors que le ravitaillement par camion ne dépasse pas 10 tonnes par heure. Si les travaux de fondations sont faits à la pelle et la pioche, des wagonnets Decauville (fig. 4) circulent sur des voies de 0,60 m pour l'évacuation des déblais. Les matériaux (aciers, ciments...) font l'objet de marchés de fournitures passés à l'échelon national et sont acheminés

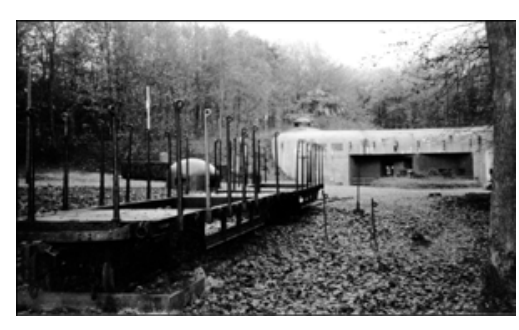

Figure 4 : Fort de Schoenbourg, wagon de transport de munitions sur la section de ligne reconstituée. Cl. J. Forthoffer, nov. 2000 par wagons dans les gares de transbordement de Soultz-sousForêt et Hunspach. Après stockage, ils sont repris par les wagonnets pour leur livraison terminale. Ainsi nait un réseau de chemin de fer à voie étroite de près de $70 \mathrm{~km}$ alors que l'avantprojet de 1928 en prévoyait $120 \mathrm{~km}$. Il emprunte le plus souvent les bas-côté de la route, mais nécessite également la construction d'ouvrages d'art. Le passage supérieur qui enjambe la ligne Haguenau-Niederbronn et la route nationale 62 à la hauteur de l'actuelle maison forestière de la Zinzel en témoigne encore aujourd'hui. L'étendue des gros ouvrages a entrainé l'installation de voies ferrées avec traction électrique (locotracteur Vetra ou SW de 5,3 tonnes) pour réaliser les transports intérieurs. Il autorise le ravitaillement direct en munitions depuis le dépôt de Neubourg. A noter que, dès 1940, les Allemands, ayant annexé l'Alsace et la Lorraine, réutilisent la voie de 60 et l'ouvrage du Hochwald, comme d'autres, pour y installer une usine d'armement. Puis, après guerre, les ouvrages furent réparés et entretenus en raison de la guerre froide et des tensions avec le bloc soviétique. Les trains furent maintenus en état de marche jusqu'au début des années 1970... 


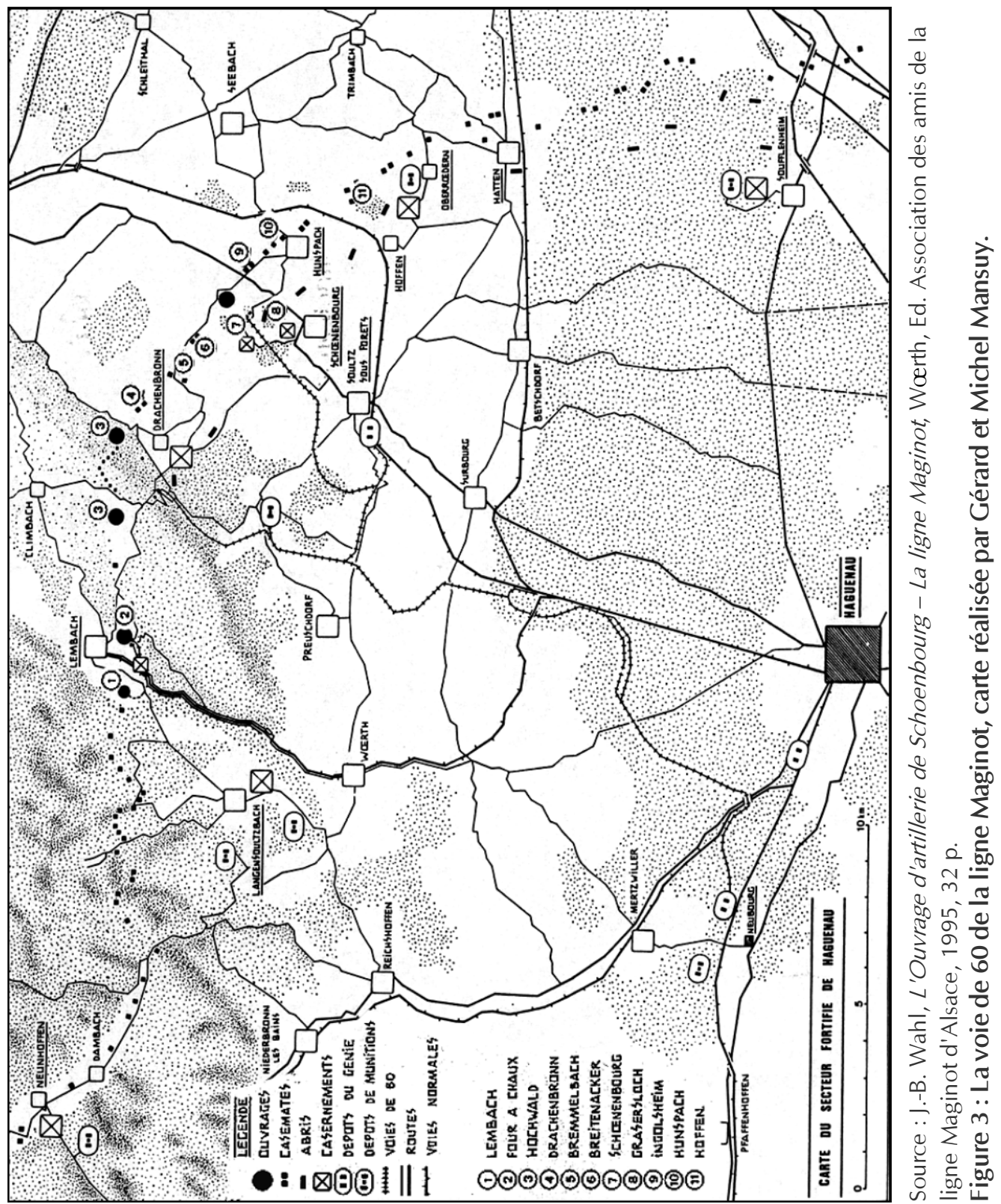


A la veille de la Seconde Guerre mondiale, d'après le bilan dressé ci-dessous (tabl. 1), le réseau des chemins de fer secondaires a été fortement réduit.

\begin{tabular}{|l|l|c|}
\hline \multicolumn{1}{|c|}{ Compagnie } & \multicolumn{1}{|c|}{ Ligne } & $\begin{array}{c}\text { Longueur } \\
(\mathbf{k m})\end{array}$ \\
\hline $\begin{array}{l}\text { Administration des chemins de fer } \\
\text { d'Alsace }\end{array}$ & Colmar-Markolsheim & 7 \\
\hline $\begin{array}{l}\text { Administration des chemins de fer } \\
\text { d'Alsace-Lorraine }\end{array}$ & Lutzelbourg-Drulingen & 12 \\
\hline $\begin{array}{l}\text { Chemin de fer de la Vallée de } \\
\text { Kaisersberg (régie } \\
\text { départementale) }\end{array}$ & Colmar-Lapoutroie & 21 \\
\cline { 2 - 3 } & Colmar-Wintzenheim & 5 \\
\hline \multirow{2}{*}{$\begin{array}{l}\text { Compagnie des tramways } \\
\text { strasbourgeois }\end{array}$} & Graffenstaden-Markolshei & 54 \\
\cline { 2 - 3 } & Strasbourg-Treuchtershei & 15 \\
\cline { 2 - 3 } & Oberhausbergen-Westhoff & 21 \\
\cline { 2 - 3 } & Rosheim-Saint Nabor & 12 \\
\hline $\begin{array}{l}\text { Compagnie des tramways de } \\
\text { Mulhouse }\end{array}$ & Réseau urbain & 20 \\
\hline $\begin{array}{l}\text { Compagnie des tramways de } \\
\text { Mulhouse-Ensisheim-Wittenheim }\end{array}$ & Mulhouse-Ensisheim & 15 \\
\cline { 2 - 3 } Société du tramway de Ribeauvillé & Mulhouse-Wittenheim & 8 \\
\hline Société d'électricité de Turckheim & $\begin{array}{l}\text { Ribeauvillé } \\
\text { gare-Ribeauvillé ville }\end{array}$ & 4 \\
\hline Régie municipale de Colmar & Turckeim-Trois Epis & 9 \\
\hline Total & Réseau urbain & 5 \\
\hline
\end{tabular}

Source : statistiques du ministère des Travaux publics pour 1927 et 1939

Tableau 1 : Chemins de fer secondaires d'intérêt général et d'intérêt local (départementaux et tramways) en Alsace en 1939.

\section{De l'après-guerre au renouveau des chemins de fer}

Dès le 10 mai 1940, la Lorraine est la première terre française directement menacée par la Wehrmacht. A la libération les destructions ont été telles dans cette région que le département du Bas-Rhin compte parmi les plus sinistrés du point de vue ferroviaire.

Le rétablissement des communications ferroviaires exige travail et imagination et s'avère plus long que prévu sur le réseau A.L. Si les convois militaires peuvent passer assez vite sur des voies souvent réparées avec des moyens de fortune, il faut attendre le 30 avril 1945 pour se rendre en train régulier de Paris à Metz et Strasbourg et le 7 mai pour atteindre Mulhouse. 
Après guerre, les petits réseaux indépendants ou les antennes ayant leur origine dans une gare du grand réseau national ont presque tous disparu. A cela deux raisons majeures : la première est la conséquence directe de la guerre 1939-1945 (destruction de la voie ou des ouvrages d'art) ; la seconde est la fermeture des sections de lignes vétustes ou ne correspondant plus aux conditions économiques.

Prenons un exemple : le chemin de fer privé de la raffinerie de Merckwiller Pechelbronn est créée en 1889. La ligne de chemin de fer à voie normale, longue de près de $5 \mathrm{~km}$, relie la raffinerie à la gare de Soultz-sous-Forêts (ligne de Strasbourg à Wissembourg). Dans un premier temps, ce sont les produits finis : essence, pétrole, gasoil, huile, paraffine qui sont acheminés vers les lieux de consommation. Une voiture de voyageurs rattachée au train permet aux salariés comme aux villageois de faire le trajet gratuitement. Après la Seconde Guerre mondiale, le pétrole brut en provenance de Bakou est acheminé par fer depuis le port de Strasbourg pour être raffiné à Pechelbronn. Mais au début des années soixante, l'activité de l'entreprise n'est plus compétitive et la ligne est fermée. Malheureusement ce sort fut réservé à de nombreux chemins de fer privés.

Les trains des lignes secondaires, ne correspondant plus aux besoins des voyageurs, reçurent des sobriquets caustiques, voire médisants, mais qui illustraient leur qualités anecdotiques. Souvent avec nostalgie, pour évoquer leur lenteur, les trains étaient appelés «tacot», « tortillard », « brouette », «Ziggel » ou « Bahnel » pour petit train... A Ingwiller, le train dont les wagons se fermaient ou s'ouvraient à l'aide d'un loquet avait gagné le sobriquet « d'Schlembe » (le loquet). Mais le substantif « Kerbsbich », « ventre de citrouille », était également attesté. Les habitants de la vallée de Kaysersberg avaient baptisé le train «Bimmelbanala » (petit train à cloche), «Halfdrucka » (aidez, poussez) voire « Kaskischt» (caisse à fromage).

Il aura fallu attendre la loi d'orientation sur les transports intérieurs (LOTI) qui ouvre en 1982 aux conseils régionaux les possibilités d'organiser les transports ferroviaires régionaux pour connaître le renouveau. La Région Alsace établit « un plan régional de transports", donne naissance au Transport Express Régional (TER) - nouveau concept multimodal de transport - et signe sa première convention avec la SNCF en 1985. Le partenariat Région Alsace-SNCF a été concrétisé par la naissance en septembre 1991 du TER 200, première desserte régionale à grande vitesse en France et même en Europe qui relie à 200 $\mathrm{km} / \mathrm{h}$ les grandes agglomérations alsaciennes : Saverne, Strasbourg, Sélestat, Colmar, Mulhouse et Bâle. 
L'apparition du schéma régional des transports (1994), puis l'expérimentation de la régionalisation (1997) menée par la Région Alsace et sous l'impulsion du sénateur Haenel conduisent au renouveau du transport régional. La réactivation de certaines lignes, comme la réouverture de la ligne Wissembourg-Winden en $1997^{4}$ (fig. 5), et des actions innovantes, comme la création de comités de lignes, en font une référence. Les projets périurbains des trams-trains de Mulhouse (fin 2005) et de Strasbourg (2006) marquent une nouvelle étape du développement des transports de proximité.

Parmi les activités de loisirs, les chemins de fer touristiques suscitent un grand intérêt auprès d'un large public en quête de nature et d'insolite. Au-delà, des passionnés, des collectionneurs se retrouvent dans des associations ${ }^{5}$, des expositions ou autour d'un projet de conservation de matériel ou de voies auxquels ils redonnent une nouvelle vie. Ils contribuent ainsi au développement du tissu socio-économique.

Le chemin de fer forestier d'Abreschviller a été créé en 1884 pour alimenter la scierie et permettre de transporter les bûcherons. Suite à plusieurs tempêtes $(1892,1902)$ il a connu une extension pour atteindre $73 \mathrm{~km}$ en 1918. L'association est créée en 1967 et, avec la signature d'une convention avec l'O.N.F., l'exploitation touristique peut démarrer l'année suivante. La ligne à voie de $0,70 \mathrm{~m}$ longue de $6 \mathrm{~km}$ relie Abreschviller à Grand-Soldat (fig. 6) avec une traction vapeur et diesel, et attire près de 10000 touristes pour la première saison.

Le chemin de fer de la vallée de la Doller (C.F.T.V.D.) est créé sur une ligne déclassée en 1973 où le trafic voyageurs a cessé en 1967. Les premiers trains touristiques circulent en 1976 sur une voie à écartement normal entre Cernay-St-André (Haut-Rhin) et Sentheim. Le parcours de $14 \mathrm{~km}$ invite à la découverte de l'écosystème naturel riche en faune et flore du piémont de la Doller. Pour des raisons de sécurité, depuis 1997 le train ne franchit plus la RN 66 pour se rendre à la gare SNCF de Cernay. Avec ces circulations et des expositions à la gare de Sentheim, il contribue à l'animation de la vallée en période estivale.

Le chemin de fer touristique du Rhin (C.F.T.R.) : la ligne appartient à la chambre de commerce et d'industrie de Colmar et dessert en semaine la zone portuaire de Neuf-Brisach (Haut-Rhin). Depuis 1983, le départ est donné en gare de Vogelsheim, la ligne longe le Rhin et traverse la forêt rhénane pour conduire, après un trajet de $9 \mathrm{~km}$, les passagers à l'embarcadère Sans-Soucis. La gare de Vogelsheim, anciennement NeufBrisach, témoigne de l'architecture des gares d'Alsace-Lorraine. Le dépôt dispose d'une riche collection de machines à vapeur. 


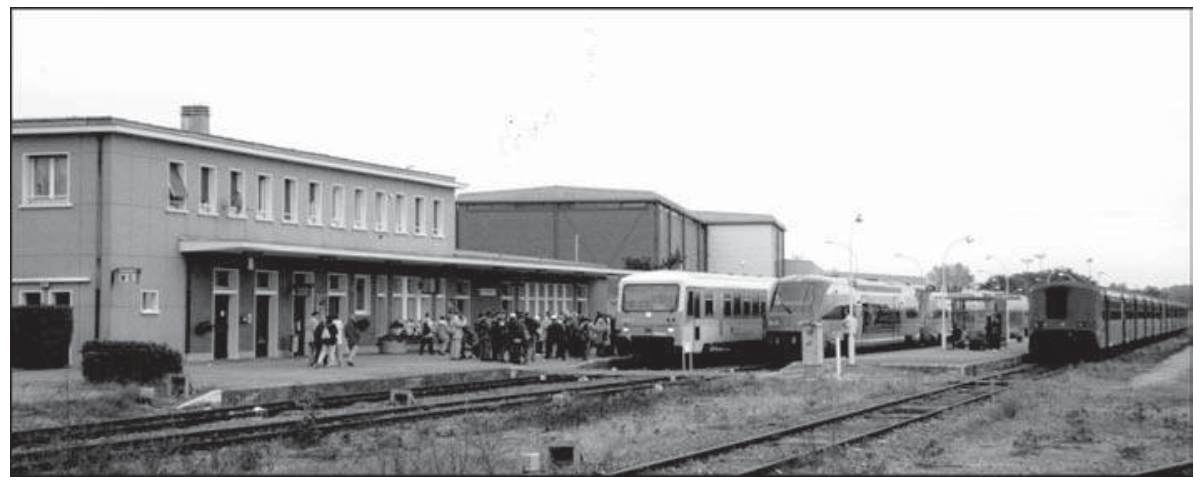

Figure 5 : La gare de Wissembourg, un dimanche soir (17 h) en octobre 2000. Des touristes allemands sur le quai A s'apprêtent à monter dans l'automoteur VT 628. Les étudiants sur le quai $B$ attendent la mise en place de l'automoteur TER. Au quai C, la rame RRR est garée. Cl. J. Forthoffer

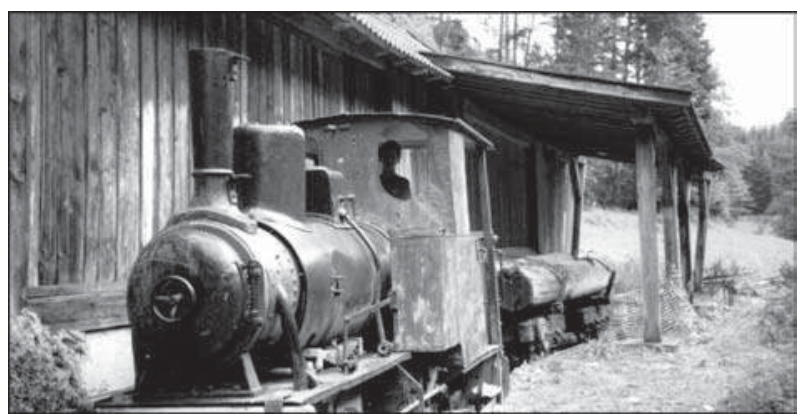

Figure 6 : Abreschviller. Scierie du Grand-Soldat. Machine à vapeur et wagonnet chargé de bois en grumes.

Cl. et coll. J. Forthoffer

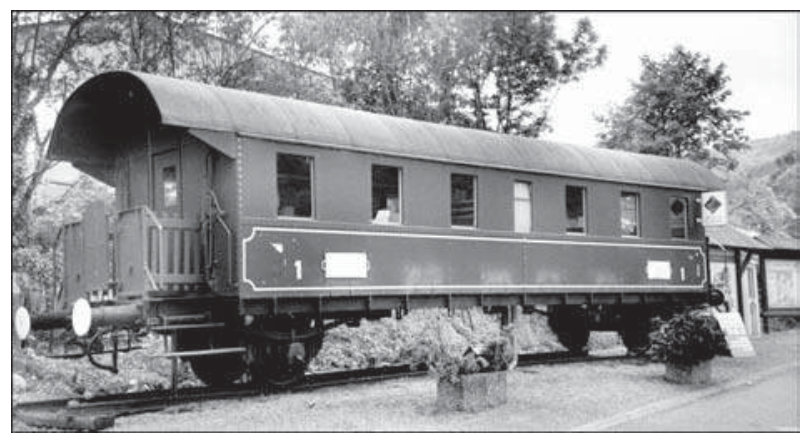

Figure 7 : L'office de tourisme de la vallée verte logé dans une voiture de chemin de fer, route de l'Europe dans la vallée de Kaysersberg, près de l'ancienne gare de Hachinette, en souvenir de l'ancienne ligne.

Cl. J. Forthoffer, mai 2001 
Cependant, aujourd'hui, la plupart des chemins de fer secondaires n'ont laissé que quelques rares témoignages comme les bâtiments de gares, des wagons utilisés comme office de tourisme. Les autres vestiges ne sont que des traces de plates-formes de voies qui se distinguent ici et là dans le paysage...

L'idée de créer un Musée français du Chemin de fer à Mulhouse remonte aux années d'après-guerre. Elle a séduit la chambre de commerce et d'industrie de Mulhouse dès 1964. Il est normal de trouver des musées techniques dans cette ville d'ancienne tradition industrielle. Sur le plan historique, il faut souligner que ce sont les industriels mulhousiens qui, dès 1839, ont construit l'une des plus anciennes voies ferrées françaises entre Mulhouse et Thann. De plus, la plus importante usine mulhousienne de l'époque, les Établissements André Koechlin et Cie, devenu Société alsacienne de construction mécanique de Mulhouse, a sorti en 1885 la première locomotive 701 Nord. La chambre de commerce s'est associée au comité de la Société industrielle de Mulhouse pour étudier les conditions de l'implantation du musée. En l'attendant, la gare du nord de Mulhouse présente à partir de 1975 une douzaine de locomotives et quelques wagons qui attirent près de 60000 visiteurs. En cette année 2001, le musée fête son $30^{\mathrm{e}}$ anniversaire et il a considérablement enrichi sa collection qui compte une centaine de machines. Il projette d'importants travaux de rénovation et d'extension pour la mise en valeur de son patrimoine.

\section{Conclusion}

A l'origine, la construction du réseau ferré des campagnes a été une initiative des industriels et avait comme vocation première le transport de marchandises : approvisionnements de matières premières et expéditions des produits finis.

Aujourd'hui, nous notons une réorientation du réseau ferré vers les zones urbaines et le transport de personnes. Il répond à un accroissement considérable du nombre de déplacements au quotidien, en particulier pour le travail, les études et les loisirs. Confrontés aux effets négatifs d'un développement rapide, les grandes agglomérations de Mulhouse et de Strasbourg optent à nouveau pour un tramway. Ce dernier est projeté non plus en étoile, mais interconnecté au réseau ferré des transports régionaux de voyageurs, à l'image de celui qui a fait ses preuves en Allemagne, à Karlsruhe et à Saarbrücken et est appelé plus communément « tram-train ». Cependant le réseau ferré, en Alsace, reste très éloigné du maillage qu'il avait atteint au début du siècle, l'industrie automobile n'étant pas étrangère à ce phénomène. 
Par ailleurs, l'augmentation du temps consacré aux loisirs et le désir de conserver le patrimoine industriel favorisent le développement des trains touristiques. La mise en service, à l'Écomusée d'Alsace d'Ungersheim, d'un train navette à l'été 2001 avec la reconstruction à l'identique de la gare de Bollwiller et la construction d'une voie longue de $600 \mathrm{~m}$ pour rejoindre la mine de potasse (Carreau Rodolphe) en est un exemple. Le chemin de fer est donc à nouveau un moteur de l'économie régionale, avec la régionalisation en marche, mais également par les attraits touristiques du passé (fig. 7).

\section{Notes}

1- Outres les ouvrages cités dans les notes qui suivent, cette contribution s'appuie sur : - Archives:

Archives départementales du Bas-Rhin, du Haut-Rhin et Archives du Land BadenWürttemberg.

- Sources imprimées en langues allemande:

«Die Wirtchaftliche Entwicklung Elsas-Lothringen 1871-1918», in A. Mündel, Streifzüge und Rastorte im Reichslande und angrenzenden Gebieten, Heft 1 Die Strassenbahn Strassburg-Markolsheim, Strasburg, 1887, pp. 519-545.

- Ourrages de référence:

- J. Coumes, Les Chemins de fer vicinaux dans le département du Bas-Rhin, Paris et Strasbourg, 1865 ;

- R. Courant, Le Temps des tramways, Menton, Les éditions du Cabri, 1982, 192 p. ; - A. Gibert, J. Bassaudo, Trains oubliés, vol. 1, L'Alsace-Lorraine - L'Est, Menton, Les éditions du Cabri, 1981, 175 p. ;

- J. Michel, Les Chemins de fer d'intérêt Local, Paris, 1866 ; C. Lamboley, Strasbourg Tramway des Vosges au Pays de Bade, Strasbourg, Ed. Contades, 1990, 164 p. ;

- J.-M. Dupuy, J. Buchmann, B. Mayer, L'Encyclopédie des chemins de fer d'AlsaceLorraine, Béthune, Locorevue, 1998, 329 p.

2- Extrait du Procès-verbal des délibérations, session de 1858, séance du 27 août, Chemins de grande communication - réseau vicinal de voies ferrées : Rapport Migneret, Conseil général du Bas-Rhin, 1858, 31 p.

3- Jean-Baptiste Migneret, Discours prononcé à Wasselonne à l'inauguration des chemins de fer de Strasbourg à Molsheim, Barr et Wasselonne, le 25 septembre 1864, Strasbourg, Imprimerie Berger Levrault, 11 p.

4- Cf. Joël Forthoffer, «Les liaisons ferroviaires transfrontalières de proximité en Alsace » in «Les chemins de fer en temps de concurrence : choix du XIX siècle et débats actuels ", actes du $6^{\circ}$ colloque de l'AHICF, Revne d'histoire des chemins de fer, 16-17 (1997), pp. 128-134.

5- Source : visite des lieux, entretiens ainsi que la consultation du site de la Fédération des amis des chemins de fer secondaires (www.trains-fr.org). 


\section{Annexe}

\section{Chronologie des lignes de chemins de fer d'Alsace}

Les tableaux ci-dessous présentent la chronologie de l'ouverture des lignes et renseignent sur l'évolution des réseaux.

\section{Des origines à 1870}

\begin{tabular}{|c|c|c|}
\hline Création & Section de ligne & $\begin{array}{c}\text { Longueur } \\
\text { (en } \\
\text { kilomètres) }\end{array}$ \\
\hline $1 / 09 / 1839$ & Mulhouse - Thann & 20 \\
\hline $18 / 10 / 1840$ & Benfeld - Colmar & 39 \\
\hline $25 / 10 / 1840$ & Mulhouse - Saint-Louis & 28 \\
\hline $1 / 05 / 1841$ & Koenigshoffen (Strasbourg) - Benfeld & 26 \\
\hline $15 / 08 / 1841$ & Colmar - Lutterbach & 37 \\
\hline $26 / 03 / 1844$ & Strasbourg (extra-muros) - Koenigshoffen & 2 \\
\hline $16 / 06 / 1844$ & Saint-Louis - Frontière suisse & 1 \\
\hline $11 / 12 / 1844$ & Fontière suisse - Bâle (ancienne gare) & 1 \\
\hline $29 / 05 / 1851$ & Koenigshoffen - Strasbourg (intra-muros) & 1 \\
\hline $29 / 05 / 1851$ & Strasbourg - Reding - Sarrebourg & 70 \\
\hline $18 / 07 / 1855$ & Vendenheim - Haguenau & 23 \\
\hline $23 / 10 / 1855$ & Haguenau - Wissembourg & 34 \\
\hline $15 / 10 / 1857$ & Mulhouse - Dannemarie & 25 \\
\hline $15 / 02 / 1858$ & Dannemarie - Montreux-Vieux & 14 \\
\hline $11 / 05 / 1861$ & Strasbourg - Kehl & 8 \\
\hline $25 / 11 / 1863$ & Thann - Wesserling & 13 \\
\hline $29 / 09 / 1864$ & Strasbourg - Molsheim -Barr & 34 \\
\hline $15 / 12 / 1864$ & Molsheim - Mutzig & 1 \\
\hline $15 / 12 / 1864$ & Molsheim - Wasselonne & 17 \\
\hline $19 / 12 / 1864$ & Haguerau - Niederbronn & 20 \\
\hline $29 / 12 / 1864$ & Sélestat - Sainte-Marie-aux-Mines & 22 \\
\hline $3 / 12 / 1868$ & Colmar - Munster & 19 \\
\hline $30 / 06 / 1869$ & Cernay - Sentheim & 14 \\
\hline $8 / 12 / 1869$ & Nierderbronn - Sarreguemines & 63 \\
\hline $5 / 02 / 1870$ & Bollwiller - Gebwiller & 7 \\
\hline
\end{tabular}


Annexion allemande 1870 - 1918

\begin{tabular}{|c|c|c|}
\hline Création & Section de ligne & $\begin{array}{l}\text { Longueur } \\
\text { (en } \\
\text { kilomètres) }\end{array}$ \\
\hline $25 / 07 / 1876$ & Strasbourg - Lauterbourg & 57 \\
\hline $1 / 08 / 1877$ & Sélestat - Barr & 18 \\
\hline $1 / 08 / 1877$ & Wasselone - Saverne & 18 \\
\hline $15 / 10 / 1877$ & Steinbourg - Bouxwiller & 13 \\
\hline $15 / 10 / 1877$ & Mutzig - Rothau & 23 \\
\hline $5 / 01 / 1878$ & Colmar - Neuf-Brisach (Alt Breisach) & 21 \\
\hline $6 / 02 / 1878$ & Mulhouse - Neuenbourg - Rhin & 18 \\
\hline $11 / 02 / 1878$ & Saint-Louis - Rhin & 4 \\
\hline $1 / 11 / 1881$ & Bouxwiller - Haguenau & 22 \\
\hline $15 / 08 / 1883$ & $\begin{array}{l}\text { Strasbourg (nouvelle-gare) - } \\
\text { Koenigshoffen }\end{array}$ & l \\
\hline $1 / 09 / 1883$ & Lutzelbourg - Phalsbourg & 6 \\
\hline $1 / 09 / 1884$ & Sentheim - Masevaux & 5 \\
\hline $1 / 11 / 1884$ & Lauterbourg - Rhin & 2 \\
\hline $15 / 12 / 1884$ & Guebwiller - Lautenbach & 6 \\
\hline $1 / 11 / 1885$ & Lutterbach - Mulhouse & 7 \\
\hline $1 / 12 / 1885$ & Colmar - Lapoutroie & l \\
\hline $1 / 12 / 1885$ & Colmar - Horbourg & 1 \\
\hline $16 / 12 / 1889$ & Bouxwiller - Ingwiller & 7 \\
\hline $1 / 10 / 1890$ & Rothau - Saales & 17 \\
\hline $3 / 11 / 1890$ & Horbourg - Marckolsheim & 22 \\
\hline $1 / 10 / 1891$ & Val de Villé - Villé & 9 \\
\hline $1 / 12 / 1891$ & Walbourg - Woerth & 9 \\
\hline $4 / 01 / 1892$ & Altkirch - Ferrette & 22 \\
\hline $4 / 01 / 1892$ & Sarrebourg - Abreschwiller & 16 \\
\hline $1 / 06 / 1892$ & Sarrebourg - Vallerysthal & 9 \\
\hline $1 / 11 / 1893$ & Munster - Metzeral & 5 \\
\hline $1 / 11 / 1893$ & Mertzwiller - Seltz & 35 \\
\hline $1 / 05 / 1895$ & Mommenheim (Strasbourg) - Kalhausen & 69 \\
\hline $1 / 05 / 1895$ & Haguenau - Roppenheim - Rhin & 29 \\
\hline $21 / 07 / 1897$ & Wingen-sur-Moder - Saint-Louis-les-Bitche & I \\
\hline $1 / 08 / 1899$ & Woerth - Lembach & 8 \\
\hline
\end{tabular}




\begin{tabular}{|c|l|c|}
\hline Création & \multicolumn{1}{|c|}{ Section de ligne } & $\begin{array}{c}\text { Longueur } \\
\text { (en } \\
\text { kilomètres) }\end{array}$ \\
\hline $1 / 07 / 1900$ & Wissembourg - Lauterbourg & $/$ \\
\hline $24 / 10 / 1901$ & Colmar - Ensisheim - Bollwiller & 35 \\
\hline $24 / 06 / 1902$ & Frontière suisse - Bâle (nouvelle gare) & 4 \\
\hline $2 / 01 / 1905$ & Wesserling - Kruth & $/$ \\
\hline $30 / 10 / 1909$ & Sélestat - Sundhouse & $/$ \\
\hline $1 / 10 / 1910$ & Dannemarie - Pfetterhouse & $/$ \\
\hline $1 / 05 / 1913$ & Drulingen - Diemeringen & $/$ \\
\hline
\end{tabular}

L'entre-deux-guerres, 1918 - 1939

\begin{tabular}{|c|l|}
\hline Création & \multicolumn{1}{c|}{ Section de ligne } \\
\hline $1 / 03 / 1920$ & Waldighoffen - Saint-Louis \\
\hline $1 / 07 / 1922$ & Schalbach - Drulingen \\
\hline $1 / 04 / 1923$ & Neuf-Brisach - Bantzenheim \\
\hline $21 / 10 / 1928$ & Saales - Saint-Dié \\
\hline $3 / 12 / 1936$ & Réding - Schalbach \\
\hline $8 / 08 / 1937$ & $\begin{array}{l}\text { Sainte-Marie-aux-Mines - Lesseux- } \\
\text { Frapelle }\end{array}$ \\
\hline
\end{tabular}

Chemins de fer industriels privés, tramways et lignes touristiques

\begin{tabular}{|c|l|l|c|}
\hline Création & \multicolumn{1}{|c|}{ Exploitant } & \multicolumn{1}{|c|}{ Section de ligne } & $\begin{array}{c}\text { Fermeture } \\
\text { (V : voyageur, } \\
\text { F : fret) }\end{array}$ \\
\hline 1879 & $\begin{array}{l}\text { Rappolsweiler } \\
\text { Strassenbahn } \\
\text { Aktiengesselschaft (RSB) }\end{array}$ & $\begin{array}{l}\text { Ribeauvillé Gare - } \\
\text { Ribeauvillé Ville }\end{array}$ & 1938 \\
\hline 1883 & $\begin{array}{l}\text { Pfalzburger Strassenbahn } \\
\text { Gesellschaft (Pf. Str. B.) }\end{array}$ & $\begin{array}{l}\text { Lutzelbourg - } \\
\text { Vilsberg }\end{array}$ & V 1949, F 1953 \\
\hline 1883 & (Pf. Str. B.) & $\begin{array}{l}\text { Maisons-Rouges - } \\
\text { Phalsbourg }\end{array}$ & V 1949, F 1953 \\
\hline 1885 & $\begin{array}{l}\text { Kaysersberger Thalbahn } \\
\text { (K. T.B.) }\end{array}$ & Colmar - Lapoutroie & 1944 \\
\hline 1885 & (K. T.B. ) & Colmar - Hohbourg & V 1937, F 1944 \\
\hline
\end{tabular}




\begin{tabular}{|c|l|l|c|}
\hline Création & \multicolumn{1}{|c|}{ Exploitant } & \multicolumn{1}{|c|}{ Section de ligne } & \multicolumn{1}{c|}{$\begin{array}{c}\text { Fermeture } \\
\text { (V : voyageur, } \\
\text { F : fret })\end{array}$} \\
\hline 1885 & (K. T.B. ) & $\begin{array}{l}\text { Colmar - } \\
\text { Wintzenheim }\end{array}$ & V 1960, F 1934 \\
\hline 1886 & $\begin{array}{l}\text { Strasburger Pferde } \\
\text { Eisenbahn Gesellschaft }\end{array}$ & $\begin{array}{l}\text { Strasbourg - } \\
\text { Boofzheim }\end{array}$ & V 1955, F 1957 \\
\hline 1886 & & $\begin{array}{l}\text { Boofzheim - } \\
\text { Marckolsheim }\end{array}$ & V 1945, F 1950 \\
\hline 1886 & & Boofzheim Rhinau & V 1955, F 1957 \\
\hline 1887 & & $\begin{array}{l}\text { Strasbourg - } \\
\text { Truchtersheim }\end{array}$ & V 1953, F 1956 \\
\hline 1889 & & Erstein & V 1955, F 1920 \\
\hline 1869 & De Dietrich & $\begin{array}{l}\text { Bannstein - } \\
\text { Mouterhouse }\end{array}$ & $\begin{array}{l}\text { F 1955 } \\
\text { Peultz - }\end{array}$ \\
\hline 1889 & $\begin{array}{l}\text { Pechelbronn } \\
\text { Oelberwerke }\end{array}$ & & \\
\hline
\end{tabular}

